\title{
INTERFACE ENTRE REGULAÇÃO EM SAÚDE E EQUIDADE: REVISÃO INTEGRATIVA DA LITERATURA*
}

\author{
Caroline Cechinel Peiter ${ }^{1}$, Gabriela Marcellino de Melo Lanzoni² ${ }^{2}$ Walter Ferreira de Oliveira ${ }^{3}$
}

\begin{abstract}
RESUMO: Este artigo tem por objetivo apresentar a interface entre a Regulação em Saúde e o princípio da equidade segundo a literatura científica da área de saúde, de 2008 a 2015. Trata-se de uma revisão integrativa da literatura, a partir de duas bases de dados, realizada em junho de 2016. Foram analisados 15 estudos que atenderam aos critérios de inclusão. A síntese do conhecimento foi feita por duas categorias de análise: 1) "Equidade e Acesso" e 2) "Reforma de sistemas de saúde". A Regulação em Saúde é compreendida como importante ferramenta para promoção da equidade. Os serviços de saúde devem priorizar o atendimento de usuários com maiores riscos, permitindo que todos tenham adequados níveis de saúde, a partir de ofertas de serviços distintos, segundo as necessidades individuais. Temas como equidade, acesso e financiamento foram pontos fortes considerados nos processos de reforma dos sistemas de saúde.

DESCRITORES: Regulação e fiscalização em saúde; Equidade; Saúde pública; Gestão em saúde.
\end{abstract}

\section{INTERFACE BETWEEN HEALTHCARE REGULATION AND EQUITY: AN INTEGRATIVE REVIEW OF THE LITERATURE}

\begin{abstract}
This article aims to present the interface between Healthcare Regulation and the principle of equity, according to the scientific literature from the area of health, from 2008 to 2015. It is an integrative review of the literature, based on two databases, undertaken in June 2016. A total of 15 studies which met the inclusion criteria were analyzed. The knowledge was summarized in two categories of analysis: 1) "Equity and Access" and 2) "Reform of health systems". Healthcare Regulation is understood as an important instrument for promoting equity. The health services must prioritize the attendance of service users with greater risks, allowing all to have adequate levels of health, based on the offering of distinct services, according to individual needs. Issues such as equity, access and financing were strong points considered in the processes of reform of health systems.

DESCRIPTORS: Health Care Coordination and Monitoring; Equity; Public health; Health management.
\end{abstract}

\section{INTERRELACIÓN ENTRE REGULACIÓN EN SALUD Y EQUIDAD: REVISIÓN INTEGRATIVA DE LA LITERATURA}

RESUMEN: Artículo con el objetivo de presentar la interrelación entre la Legislación de Salud y el principio de equidad en el área de salud según la literatura científica, de 2008 a 2015. Revisión integrativa de la literatura, partiéndose de dos bases de datos, realizada en junio de 2016. Fueron analizados 15 estudios que atendieron los criterios de inclusión. La síntesis del conocimiento se ejecutó en dos categorías de análisis: 1) "Equidad y Acceso" y 2) "Reforma de sistemas de salud". La Legislación en Salud constituye una importante herramienta para la promoción de la equidad. Los servicios de salud deben priorizar la atención de usuarios con mayores riesgos, permitiendo que todos cuenten con niveles de salud adecuados, a partir de ofertas de servicios distintos, según las necesidades individuales. Temas como equidad, acceso y financiamiento fueron puntos fuertes considerados en los procesos de reforma de los sistemas de salud.

DESCRIPTORES: Regulación y Fiscalización en Salud; Equidad; Salud Pública; Gestión en Salud.

*Artigo extraído da dissertação de mestrado intitulada: "Regulação em saúde: um diálogo com o princípio da equidade". Universidade Federal de Santa Catarina, 2014.

${ }^{1}$ Enfermeira. Doutoranda em Enfermagem. Universidade Federal de Santa Catarina. Florianópolis, SC, Brasil.

${ }^{2}$ Enfermeira. Doutora em Enfermagem. Docente de Enfermagem da Universidade Federal de Santa Catarina. Florianópolis, SC, Brasil.

${ }^{3}$ Médico. Doutor em Filosofia, Fundamentos Sociais e Filosóficos da Educação. Docente da Universidade Federal de Santa Catarina. Florianópolis, SC, Brasil.

Autor Correspondente:

Recebido: 21/06/2016

Caroline Cechinel Peiter

Finalizado: $23 / 02 / 2017$

Universidade Federal de Santa Catarina.

Campus Reitor João David Ferreira Lima, s/n. - 88.040-900 - Florianópolis, SC, Brasil

E-mail: carolcechinel@gmail.com 


\section{INTRODUÇÃO}

Cada vez mais, o tema Regulação em Saúde vem ganhando espaço nas discussões acerca da gestão em saúde, devido ao seu potencial de contribuição para o setor ${ }^{(1)}$. No Brasil, foi normatizada em 2008, pela Política Nacional de Regulação do Sistema Único de Saúde (SUS), dividindo a Regulação em Saúde em três funções principais: Regulação de Sistemas de Saúde; Regulação da Atenção à Saúde; Regulação do Acesso à Assistência ${ }^{(2)}$.

A Regulação de Sistemas de Saúde se refere às ações de monitoramento, controle, avaliação, auditoria e vigilância, enquanto a Regulação da Atenção à Saúde diz respeito ao controle da oferta de serviços, bem como tem por finalidade a adequada prestação de serviços à população, por meio de prestadores públicos e privados, definindo estratégias para a Regulação do Acesso à Assistência. A terceira, Regulação do Acesso à Assistência, tem por objetivo gerenciar a priorização do acesso e dos fluxos assistenciais, abrangendo a regulação médica. Esta garante o acesso aos usuários baseado em classificação de risco e outros critérios para priorização ${ }^{(3)}$.

A classificação de risco permite que a oferta dos serviços seja direcionada aos casos prioritários, possibilitando a equidade da oferta das ações de modo a obedecer aos limites das cotas financeiras de cada município ${ }^{(4)}$. Associado a isto, o tema equidade em saúde tem sido relacionado ao conceito de necessidade. Considerando que cada indivíduo possui necessidades diferentes, e que, portanto, um tratamento igualitário de saúde não atenderia satisfatoriamente as suas demandas, é justificado o emprego do processo de Regulação da oferta para otimizar o atendimento à população e minimizar as iniquidades em saúde ${ }^{(5)}$.

O desconhecimento dos profissionais que atuam no SUS sobre essa ferramenta faz com que estes tenham uma compreensão aquém da real importância da Regulação em Saúde para o sistema, o que indica a necessidade de capacitação dos gestores acerca do tema e instrumentalização dos profissionais envolvidos para um serviço de Regulação em Saúde eficiente, capaz de proporcionar todas as facilidades a que a Regulação se propõe ${ }^{(1)}$.

Nesse sentido, questiona-se: Qual a interface entre a Regulação em Saúde e o princípio da equidade segundo a literatura científica da área da Saúde? Pautada nos aspectos apresentados, esta revisão tem por objetivo apresentar a interface entre a Regulação em Saúde e o princípio da equidade segundo a literatura científica da área de saúde, de 2008 a 2015.

\section{METODOLOGIA}

Trata-se de um estudo de abordagem quantitativa, para identificação de produções sobre o tema Regulação em Saúde e Equidade, entre 2008 e 2015. Para atingir o objetivo proposto, adotouse a revisão integrativa da literatura, que contribui para o processo de sistematização e análise dos resultados, buscando a ampliação da compreensão da temática proposta a partir da análise de estudos anteriormente publicados. O contato com o material científico publicado na área de conhecimento a ser investigada permite ao pesquisador conhecer as lacunas existentes na produção científica, o que indica a necessidade de realização de novos estudos ${ }^{(6)}$.

A pesquisa seguiu os padrões de rigor metodológico propostos para a revisão integrativa da literatura, envolvendo coleta de dados, análise e apresentação dos resultados, desde o início do estudo, a partir de um protocolo de pesquisa previamente elaborado e validado. Para tanto, foram percorridas as seis etapas sugeridas para desenvolvimento do método: 1) identificação do tema e seleção da hipótese ou questão de pesquisa para a elaboração da revisão integrativa; 2) estabelecimento de critérios para inclusão e exclusão de estudos/amostragem ou busca na literatura; 3) definição das informações a serem extraídas dos estudos selecionados/categorização dos estudos; 4) avaliação dos estudos incluídos na revisão integrativa; 5) interpretação dos resultados; e 6) apresentação da revisão/síntese do conhecimento ${ }^{(7)}$.

Na primeira etapa, formulou-se a questão norteadora: Qual a interface entre a Regulação em Saúde e o princípio da equidade segundo a literatura científica da área da Saúde? 
A segunda etapa envolveu a busca bibliográfica, que ocorreu no mês de junho de 2016 nas bases de dados, Medical Literature and Retrieval System onLine (MEDLINE) e Literatura Latino-americana e do Caribe em Ciências da Saúde (LILACS). As bases de dados foram acessadas por meio do link disponibilizado pela Biblioteca Universitária da Universidade Federal de Santa Catarina (BU/UFSC).

Foram adotados os seguintes critérios para seleção dos artigos: todas as categorias de artigo (pesquisa original, revisão de literatura, relato de experiência, editorial); artigos com resumos disponíveis para análise; publicados em todos os idiomas, entre os anos 2008 e 2015, ou seja, posterior à Política Nacional de Regulação do Sistema Único de Saúde no Brasil(2); e artigos que contivessem os seguintes descritores em ciências da saúde (DeCS) ao longo de todo o texto: Government Regulation and Equity. Foram critérios de exclusão: resenhas, anais de congresso, teses, dissertações, boletins epidemiológicos, artigos não relacionados ao objetivo deste estudo, e aqueles que não atendessem aos critérios de inclusão mencionados.

Foram localizados 54 estudos. Estes foram submetidos a uma leitura flutuante, na qual foram analisados seus títulos e resumos. Excluiu-se, desta forma, quatro documentos que não tratavam de artigo científico, e toda a produção não relacionada com o escopo do estudo, tais como artigos relacionados à regulamentação/legislação sobre práticas clínicas (oito); regulamentação ambiental (seis); regulamentação da prática profissional (cinco); reprodução humana (três); regulação de novos medicamentos (dois); regulação de alimentos (dois); gestão de recursos humanos (um); seguro saúde (um); pesquisas (um); e transplantes (um). Após esta etapa, 20 artigos foram pré-selecionados e submetidos à leitura integral. Esta análise possibilitou identificar outros cinco artigos não relacionados ao objetivo desta pesquisa. Assim, 15 foram selecionados para análise aprofundada.

Na terceira etapa, os artigos selecionados foram organizados de modo a facilitar o acesso, para posterior leitura integral e fichamento das informações relevantes. As informações presentes nestes artigos foram agrupadas em tabela do software Excel, sendo discriminados: ano de publicação, autores, título, resumo, país de origem, idioma, periódico publicado e considerações referentes ao tema.

Na quarta etapa os achados foram agrupados conforme suas semelhanças e diferenças. A quinta etapa envolveu uma análise aprofundada dos artigos, e a partir dos agrupamentos foram formadas as categorias temáticas do estudo, seguindo-se com discussão sustentada a partir da literatura pertinente. O processo foi finalizado com a sexta etapa, onde foi processada, então, a síntese do conhecimento.

\section{- RESULTADOS}

Os 15 artigos selecionados para análise estavam distribuídos em periódicos distintos, não havendo concentração relevante de estudos com a temática pesquisada em periódicos específicos. 2011 foi o ano em que houve maior número de publicações sobre o tema, um total de quatro $(26,7 \%)$, seguido, consecutivamente, de 2012 e 2015 com três estudos (20,0\%), 2014 com dois estudos (13,3\%), e 2008, 2009 e 2010 com um estudo $(6,7 \%)$.

Apenas um artigo estava disponível em português. Todos os demais estavam na língua inglesa. No que diz respeito ao país de origem, os Estados Unidos foram responsáveis pela produção de cinco estudos, sendo o mais frequente, seguido da Índia com duas publicações. África do Sul, Austrália, Países Baixos, Brasil, Argentina, Irã, Canadá e Suécia tiveram uma publicação.

Os achados foram agrupados de acordo com suas semelhanças e diferenças, ou seja, similaridade de assuntos, abordagens ou formas de tratamento. Desta análise, foram formuladas as categorias temáticas para realizar a síntese do conhecimento da revisão integrativa, mostradas a seguir: 1) Equidade e Acesso e 2) Reforma de Sistemas de Saúde.

\section{Categoria 1 - Equidade e Acesso}

As iniquidades em saúde estão relacionadas com o risco de doenças ou agravos e outras condições adversas, tais como desigualdade no acesso a cuidados de saúde. Elas afetam negativamente grupos específicos da população, socialmente desfavorecidos, definidos segundo fatores como raça/etnia/cor 
da pele, nível de escolaridade, renda, gênero, orientação sexual, idade, presença de doença, agravo ou deficiência, dentre outras características associadas à discriminação e marginalização ${ }^{(8-9)}$.

Sob influência de alguns dos determinantes citados, as condições sociais em que as pessoas nascem, vivem e trabalham são importantes para a situação de saúde do indivíduo, podendo influenciar desigualdades em saúde ${ }^{(10)}$. Como resultado de um desenvolvimento social, econômico e político nãoequânime, os grupos minoritários gerados por estes condicionantes tendem a ter piores condições de saúde, menor qualidade de vida e menos acesso a serviços de saúde ${ }^{(8,10-11)}$.

Para transpor tais dificuldades, é necessário adotar medidas estruturais para além do setor saúde, incluindo maiores investimentos em educação, habitação, emprego, renda, e acesso aos serviços de saúde. A distribuição desigual dessas condições sociais contribui para as persistentes iniquidades em saúde $^{(10-12)}$.

Os serviços de saúde devem ser pautados em atender às necessidades de usuários que estejam em maior risco de saúde e que apresentem mais fatores dificultadores do acesso. O intuito deve ser tratar desigualmente aqueles que se apresentam em situações desfavoráveis, buscando-se atingir a equidade, importante pilar de um sistema de saúde justo e eficiente ${ }^{(13)}$.

Aos profissionais de saúde, cabe pensar e agir de modo interdisciplinar e intersetorial, fortalecendo a força de trabalho e abordando os determinantes sociais que interferem no setor saúde, medidas que podem contribuir para corrigir as desigualdades e alcançar a equidade em saúde em um sistema eficiente $^{(8,12)}$.

O acesso aos serviços de saúde é conceituado como o ingresso do usuário às ações de atenção à saúde das quais necessita, respeitados o nível de atenção requerido e as limitações dos recursos disponíveis para a oferta ${ }^{(8)}$. Possibilitar este acesso de maneira integral, universal e equânime requer, ainda, a superação de desafios relativos à prestação de serviços e ao financiamento da saúde. É preciso garantir o acesso ao nível adequado e necessário ao paciente, contando-se muitas vezes com recursos limitados, especialmente quando se trata do uso de tecnologias avançadas em saúde ${ }^{(8,13-16)}$.

A disponibilização de um financiamento adequado ao subsídio dos serviços e tecnologias necessários em saúde é importante para ajustar a oferta à demanda, melhorar as condições de saúde da população e se aproximar, assim, da equidade ${ }^{(14-15)}$.

A otimização dos recursos, observada a limitação financeira do Estado, é indispensável na busca de atingir as necessidades de saúde da população. Há que racionalizar as intervenções essenciais e ao mesmo tempo possibilitar que todos desfrutem igualmente do mais elevado nível de saúde, por meio das diferentes ofertas, segundo as necessidades individuais ${ }^{(8-9,12-14,16-17)}$. O estabelecimento de um orçamento em saúde deve estar relacionado ao diagnóstico das necessidades de saúde da população(8,15).

A Atenção Primária em Saúde (APS) é evidenciada como um importante instrumento facilitador de acesso do usuário aos serviços de saúde mais adequados às suas necessidades, além de reduzir o custo dos atendimentos através da otimização dos recursos, e minimizar as desigualdades na saúde. A abordagem da APS proporciona atendimento integral do indivíduo, prevenção de doenças e agravos, promoção da saúde em nível comunitário e individual, e articulação para o desenvolvimento de ações intersetoriais para a saúde. Por meio do conhecimento da realidade de determinada população, a APS amplia o conhecimento das suas necessidades de saúde, o que permite a referência aos níveis de atenção mais complexos e ações de saúde de maior tecnologia aos casos que demonstram necessidade ${ }^{(14,18)}$.

Para tanto, os profissionais envolvidos devem estar capacitados para identificar as reais necessidades da população. A garantia de recursos humanos adequados, encorajados para uma abordagem interdisciplinar, engajados nos princípios da saúde pública, e incentivados a concentrar-se nas necessidades dos indivíduos que se encontram em maiores riscos, são maneiras de utilizar a força de trabalho como ferramenta para a abordagem dos determinantes sociais e de ampliar a visão para os caminhos para a equidade em saúde ${ }^{(18)}$. 
No contexto do acesso equânime aos serviços de saúde, alguns estudos descreveram experiências de países que ultrapassaram reformas em seus sistemas de saúde com o intuito de superar estes desafios, bem como a necessidade de reformulação de estratégias que dificultam o desenvolvimento da equidade em saúde.

As reformas nos sistemas de saúde foram abordadas como mudanças essenciais para alcance e manutenção de um sistema universal e equânime, permitindo resposta às necessidades de saúde a partir das evidências. Tais mudanças são processos constantes, que devem acompanhar a transformação das necessidades da população(9,19-20).

Por meio de debates sobre a melhor forma de providenciar a qualificação do acesso aos cuidados de saúde, foi evidenciada a eficiência da equidade na reformulação sanitária. As reformas sanitárias em questão levam a discussões sobre os temas equidade, acesso e financiamento ${ }^{(13,19-20)}$.

Para construir um sistema de saúde pautado no princípio da equidade, é necessário que o Estado em questão assuma o compromisso de atingir a igualdade entre todos os cidadãos. Estruturar uma cobertura de serviços de saúde de qualidade, com recursos adequados, é imprescindível para seu alcance $^{(19)}$.

Um sistema de saúde que funcione de forma equânime é considerado fundamental para a sociedade. No entanto, a equidade do acesso a tais serviços de saúde ainda é dificultada pelas desigualdades sociais, tais como referentes à renda, região de habitação e grau de escolaridade, condições de moradia e trabalho. Essas condições são constantemente citadas no contexto dos sistemas de saúde apresentados, o que dificulta a construção de um sistema de saúde baseado neste princípio ${ }^{(11,13,19)}$.

Os estudos analisados indicam a importância dos mecanismos de relação custo-eficiência, e do controle dos recursos financeiros ${ }^{(9,12,17,20-22)}$. Sugerem, ainda, que as novas reformas devam se concentrar no desenvolvimento de estratégias para enfrentamento dos problemas existentes na equidade, no acesso e no financiamento, qualificando a alocação e distribuição dos recursos. Evidencia-se, assim, a importância de reflexão sobre o processo de alocação e distribuição dos recursos, e priorização das ações a serem desenvolvidas ${ }^{(9,12,21-22)}$.

\section{DISCUSSÃO}

A literatura evidencia a forte relação entre as minorias sociais e a dificuldade no acesso aos serviços de saúde. Aspectos relacionados à etnia/raça/cor da pele, região de moradia, escolaridade e condições financeiras, geram barreiras econômicas, organizacionais e culturais, o que exige mais atenção a esses grupos, a fim de identificar suas necessidades de priorização devido à maior vulnerabilidade ${ }^{(23-24)}$.

O conceito de vulnerabilidade é entendido como a probabilidade de uma população ser atingida por problemas, ameaças, ou danos à saúde. Tal suscetibilidade leva à necessidade de priorização da assistência a tais eventos, promovendo, assim, o princípio da equidade ${ }^{(25)}$. A equidade é definida como a oferta de maior atenção aos indivíduos que mais a necessitam, provendo a cada um as ações de saúde segundo as suas demandas, com o intuito de superar as desigualdades existentes. Esta compreensão corrobora com o valor de justiça que está associado a este princípio, o que a diferencia do conceito de igualdade ${ }^{(26)}$.

Responder às demandas de uma população leva à necessidade de investimento de recursos financeiros. A superação do desafio de responder às demandas dos usuários utilizando os recursos que se têm disponíveis depende da eficácia e otimização dos recursos financeiros e do desenvolvimento de uma relação custo-benefício positiva ${ }^{(25)}$. O acesso às ações e serviços de saúde permite a integralidade do cuidado, por meio do fluxo do usuário pela rede de atenção à saúde, garantindo a oferta de serviços de diferentes densidades tecnológicas de acordo com sua necessidade ${ }^{(27)}$.

Outro desafio apontado é a identificação das necessidades em saúde de determinada população, o que pode dificultar uma distribuição equitativa dos recursos alocados em saúde, ou, do ponto de vista da prática profissional, limitar a decisão do médico regulador de decidir quais pacientes cujos agravos de saúde mostram-se prioritários diante de determinado contexto ${ }^{(26)}$. 
Faz-se necessária a atuação da APS no que concerne ao contato com o usuário, para que esta conheça o estado e necessidades de saúde dentro das suas condições de vida, e consiga realizar uma priorização confiável. Desta forma, a equipe de saúde consegue disponibilizar as ações de saúde de que o indivíduo demanda, encaminhando-o aos serviços de saúde de outros níveis de atenção quando julgar necessário, articulando todas as informações oportunas com a central de Regulação ${ }^{(4)}$.

A criação do SUS teve como um dos seus objetivos diminuir as desigualdades no acesso da população do país aos serviços de saúde ${ }^{(4)}$, entretanto, apesar de ser um sistema de saúde doutrinariamente equânime, encontra-se inserido em uma sociedade injusta e desigual, o que favorece um acesso seletivo e excludente ${ }^{(28)}$.

A compreensão de saúde ultrapassa o conceito da assistência médica, implica que um sistema de saúde equânime deve ser resultado de boas condições de trabalho, proteção social, remuneração adequada, educação, lazer, boas condições de moradia, levando à necessidade de mobilização transdisciplinar e

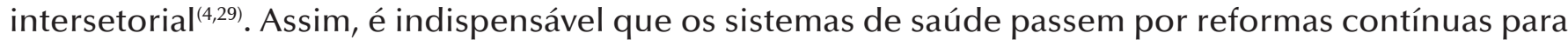
atender às mudanças nas necessidades da população e acompanhar o desenvolvimento das ciências da saúde e dos serviços públicos de saúde.

\section{CONSIDERAÇÕES FINAIS}

A Regulação em Saúde é compreendida como importante ferramenta para promoção da equidade. Vislumbrou-se que os serviços de saúde devem priorizar que todos tenham adequados níveis de saúde, a partir de ofertas de serviços distintos, segundo as necessidades individuais. É indispensável a otimização dos recursos, para que se consiga adequar oferta e demanda, tendo em vista a limitação financeira do Estado. Nesse processo, a APS foi identificada como instrumento importante para a regulação em saúde, facilitador do acesso e promotor da equidade. Temas como equidade, acesso e financiamento foram pontos fortes considerados nos processos de reforma dos sistemas de saúde.

Os achados deste estudo revelam a deficiência de artigos que abordem a Regulação em Saúde em consonância com o princípio da equidade. A dificuldade principal encontrada na etapa metodológica deste estudo se deu na inexistência de terminologia própria para o tema da pesquisa, levando a muitos estudos que não abordavam a temática. Os artigos localizados a partir da terminologia utilizada dizem respeito à regulamentação de diversas temáticas na área da saúde, não estando relacionados, necessariamente, à regulação do acesso, tema que este trabalho se propôs a pesquisar.

Um fator preocupante dentre os achados foi o baixo número de artigos brasileiros, o que indica ser oportuno o desenvolvimento científico que articule a Regulação em Saúde e o princípio da equidade, sendo proveitoso que o tema seja estruturado e desenvolvido a partir de achados científicos. A importância da Regulação em Saúde, bem como seu potencial para efetivar o exercício do princípio da equidade, sugere o investimento em novos estudos que contemplem a temática a fim de qualificar os serviços de saúde. Os resultados encontrados neste trabalho sugerem maior aprofundamento desta abordagem referente aos dois temas, de modo a identificar achados cientificamente significativos que possam qualificar o acesso equânime aos serviços de saúde e incentivar a otimização dos recursos na Saúde Pública.

\section{REFERÊNCIAS}

1. de Oliveira RR, Elias PEM. Conceitos de Regulação em Saúde no Brasil. Rev. Saúde Pública. [Internet] 2012;46(3) [acesso em 15 jun 2016]. Disponível: http://dx.doi.org/10.1590/S0034-89102012000300020.

2. Brasil. Portaria n. 1.559, de $1^{\circ}$ de agosto de 2008. Institui a Política Nacional de Regulação do Sistema Único de Saúde - SUS. Diário Oficial da República Federativa do Brasil, Brasília, 04 ago. 2008. Seção 1:48.

3. Ministério da Saúde (BR). Conselho Nacional de Secretários de Saúde. Regulação em Saúde. Brasília: CONASS; 2007.

4. Viegas SMF, Penna CMM. O SUS é universal, mas vivemos de cotas. Ciênc. saúde coletiva. [Internet] 2013;18(1) 
[acesso em 15 jun 2016]. Disponível: http://www.scielo.br/pdf/csc/v18n1/19.pdf.

5. Sancho LG, Silva NEK. Descortinando o acesso aos serviços de saúde na perspectiva da interdisciplinaridade: debate de ideias. Physis (Rio J). [Internet] 2013;23(2) [acesso em 15 jun 2016]. Disponível: http://www.scielo.br/ pdf/physis/v23n2/v23n2a04.pdf.

6. Whittemore R, Knafl K. Methodological issues in nursing research:The integrative review: updated methodology. J Adv Nurs. 2005;52(5):546-53.

7. Ganong LH. Integrative Reviews of Nursing. Rev Nurs Health. 1987;10(1):1-11.

8. Balarajan Y, Selvaraj S, Subramanian SV. Health care and equity in India. Lancet. [Internet] 2011;377(9764) [acesso em 15 jun 2016]. Disponível: http://www.thelancet.com/journals/lancet/article/PIIS0140-6736(10)61894-6/ fulltext.

9. Gordeev VS, Pavlova M, Groot W. Two decades of reforms: Appraisal of the financial reforms in the Russian public healthcare sector. Health policy. [Internet] 2011;102(2) [acesso em 15 jun 2016]. Disponível: http://www. healthpolicyjrnl.com/article/S0168-8510(10)00233-2/fulltext.

10. Starfield B. Primary care: an increasingly important contributor to effectiveness, equity, and efficiency of health services. Gac Sanit. [Internet] 2012;26(Suppl 1) [acesso em 15 jun 2016]. Disponível: http://www.lgaa.Iv/ sites/default/files/dati/Publiska_lapa/PHC\%20Barbara\%20Starfield\%202012.pdf.

11. Peters DH, Garg A, Bloom G, Walker DG, Brieger WR, Rahman MH. Poverty and access to health care in developing countries. Ann N Y Acad sci. [Internet] 2008;(1136) [acesso em 15 jun 2016]. Disponível: http:// onlinelibrary.wiley.com/doi/10.1196/annals.1425.011/pdf.

12. Sanders D, Baum FE, Benos A, Legge D. Revitalising primary healthcare requires an equitable global economic system - now more than ever. J Epidemiol Community Health. [Internet] 2011;65(8) [acesso em 15 jun 2016]. Disponível: http://jech.bmj.com/content/65/8/661.long.

13. Tataw D. The second market failure phenomenon in safety-net health systems: the case of a municipal academic medical center from 1980 to 2000. Soc Work Public Health. [Internet] 2011;26(3) [acesso em 15 jun 2016]. Disponível: http://dx.doi.org/10.1080/19371918.2011.528736.

14. Baum FE, Margaret Anaf J. Transnational corporations and health: a research agenda. Int J Health Serv. [Internet] 2015;45(2) [acesso em 15 jun 2016]. Disponível: http://joh.sagepub.com/content/45/2/353.full.pdf+html.

15. Fredriksson $M$, Blomqvist P, Winblad U. Recentralizing healthcare through evidence-based guidelines - striving for national equity in Sweden. BMC Health Serv Res. [Internet] 2014;5(14) [acesso em 15 jun 2016]. Disponível: http://bmchealthservres.biomedcentral.com/articles/10.1186/s12913-014-0509-1.

16. Garg P, Nagpal J. A review of literature to understand the complexity of equity, ethics and management for achieving public health goals in India. J Clin Diagn Res. [Internet] 2014;8(2) [acesso em 15 jun 2016]. Disponível: https://www.ncbi.nlm.nih.gov/pmc/articles/PMC3972518/.

17. Longo MC. Good practices in health care "management experimentation models": insights from an international public-private partnership on transplantation and advanced specialized therapies. Adv Health Care Manag. [Internet] 2015;(17) [acesso em 15 jun 2016]. Disponível: http://www.emeraldinsight.com/doi/abs/10.1108/ S1474-823120140000017005.

18. Castro AL, Machado CV. Primary health care policy in Brazil: notes on Federal regulation and funding. Cad. Saúde Pública. [Internet] 2010;26(4) [acesso em 15 jun 2016]. Disponível: http://www.scielo.br/pdf/csp/v26n4/12. pdf.

19. Patel V, Parikh R, Nandraj S, Balasubramaniam P, Narayan K, Paul VK, Kumar AK, et al. Assuring health coverage for all in India. Lancet. [Internet] 2015;386(10011) [acesso em 15 jun 2016]. Disponível: http://www.thelancet.com/ journals/lancet/article/PIIS0140-6736(15)00955-1/fulltext.

20. Davari M, Haycox A, Walley T. The Iranian health insurance system; past experiences, present challenges and future strategies. Iran J Public Health. [Internet] 2012;41(9) [acesso em 15 jun 2016]. Disponível: https://www.ncbi. nlm.nih.gov/pmc/articles/pmid/23193499/. 
21. Rubinstein A, Pichon-Riviere A, Augustovski F. Development and implementation of health technology assessment in Argentina: two steps forward and one step back. Intj Technol Assess Health Care. [Internet] 2009;25(Suppl 1) [acesso em 15 jun 2016]. Disponível: https://www.ncbi.nlm.nih.gov/pmc/articles/PMC3494208/.

22. Quesnel-Vallée A, Renahy E, Jenkins T, Cerigo H. Assessing barriers to health insurance and threats to equity in comparative perspective: the Health Insurance Access Database. BMC Health Serv Res. [Internet] 2012;12(107) [acessoem15jun2016].Disponível:http://bmchealthservres.biomedcentral.com/articles/10.1186/1472-6963-12-107.

23. Trad LAB, Castellanos MEP, Guimarães MCS. Acessibilidade à atenção básica a famílias negras em bairro popular de Salvador, Brasil. Rev. Saúde Pública. [Internet] 2012;46(6) [acesso em 15 jun 2016]. Disponível: http:// www.scielo.br/pdf/rsp/v46n6/10.pdf.

24. Costa SM, Abreu MHNG, Vasconcelos M, Lima RCGS, Verdi M, Ferreira EF. Desigualdades na distribuição da cárie dentária no Brasil: uma abordagem bioética. Ciênc. saúde coletiva. [Internet] 2013;18(2) [acesso em 15 jun 2016]. Disponível: http://www.scielo.br/pdf/csc/v18n2/17.pdf.

25. Soares DS, Brandão RRM, Mourão MRN, Azevedo VLF, Figueiredo AV, Trindade ES. Relevância de exames de rotina em pacientes de baixo risco submetidos a cirurgias de pequeno e médio porte. Rev. Bras. Anestesiol. [Internet] 2013;63(2) [acesso em 15 jun 2016]. Disponível: http://www.scielo.br/pdf/rba/v63n2/v63n2a07.pdf.

26. Rosas MA, Bezerra AFB, Duarte-Neto PJ. Uso das redes neurais artificiais na aplicação de metodologia para alocação de recursos da saúde. Rev. Saúde Pública. [Internet] 2013;47(1) [acesso em 15 jun 2016]. Disponível: http://www.scielo.br/pdf/rsp/v47n1/17.pdf.

27. Brondani JE, Leal FZ, Potter C, Silva RM, Noal HC, Perrando MS. Desafios da referência e contrarreferência na atenção em saúde na perspectiva dos trabalhadores. Cogitare Enferm. [Internet] 2016;21(1) [acesso em 15 jun 2016]. Disponível: http://revistas.ufpr.br/cogitare/article/view/43350/27700.

28. Assis MMA, Jesus WLA. Acesso aos serviços de saúde: abordagens, conceitos, políticas e modelo de análise. Ciênc. saúde coletiva. [Internet] 2012;17(11) [acesso em 15 jun 2016]. Disponível: http://www.scielo.br/pdf/csc/ v17n11/v17n11a02.pdf.

29. Martins MIC, Molinaro A. Reestruturação produtiva e seu impacto nas relações de trabalho nos serviços públicos de saúde no Brasil. Ciênc. saúde coletiva. [Internet] 2013;18(6) [acesso em 15 jun 2016]. Disponível: http://www.scielo.br/pdf/csc/v18n6/18.pdf. 